\title{
DEVELOPMENT OF A ROTARY TYPE
}

\section{WATER HYDRAULIC PRESSURE INTENSIFIER}

\author{
Shigeru OSHIMA*, Takuya HIRANO*, Shimpei MIYAKAWA**, and Yoshihiro OHBAYASHI** \\ * Department of Control \& Computer Engineering \\ Numazu National College of Technology \\ 3600 Ooka, Numazu, Shizuoka, 410-8501 Japan \\ (E-mail: oshima@numazu-ct.ac.jp) \\ ** Water Hydraulics System Group, Basic Technology R\&D Center \\ KYB Corporation \\ 1-12-1 Asamizodai, Sagamihara, Kanagawa, 228-0828 Japan
}

\begin{abstract}
This study concerns a new rotary type water hydraulic pressure intensifier. The object of this paper is to introduce the structure and the working principle of the pressure intensifier, and to present the fundamental characteristics by experimental results. The basic structure is similar to that of a water hydraulic planetary gear motor. Its main part consists of a stator, a rotor, nine planetary gears and two side plates. The inside of the stator is formed by a curve with 5 lobes and the outside of the rotor is formed by a curve with 4 lobes. Their curved surfaces have teeth like a gear. Nine displacement chambers are formed by the stator, rotor, planetary gears and side plates, and the each chamber's volume varies periodically when the rotor rotates. A side plate has 5 inlet distribute holes and 5 outlet distribute holes alternately located on its surface. Each displacement chamber connects to an inlet distribute hole during the volume is increasing, and it connects to an outlet distribute hole during the volume is decreasing. Now, one of the outlet distribute holes is separated from the other 4, the intensified higher pressure water is discharged from the separated distribute hole. It is found that the critical intensification ratio is about 2.5 by experimental results with a prototype.
\end{abstract}

\section{KEY WORDS}

Water hydraulics, Pressure intensifier, Rotary type, Planetary gear motor, Experimental results

\section{NOMENCLATURE}

$N:$ rotational speed of a rotor

$n$ : number of each the inlet distribute holes and the outlet distribute holes

$P_{e}:$ exhaust pressure

$P_{i}:$ intensified pressure

$P_{s}:$ supply pressure

$Q:$ flow rate

$T$ : torque

$V_{t h}:$ theoretical displacement volume

$\eta$ : total efficiency
$\eta_{T}:$ torque efficiency

$\eta_{V}:$ volumetric efficiency

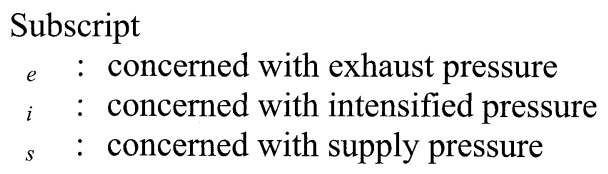

\section{INTRODUCTION}

Water hydraulic system which uses tap water as pressure medium has been known as an environmental 
friendly new fluid power drive system. It has many advantages; clean, non-toxic, non-flammable, low pressure loss, and so on [1]. It is also a big advantage that the pressure medium is easily obtained and the disposal of the waste is easy, too. They lead to decreasing of management cost.

It is considered that the water hydraulic system has many possible applications in low pressure driving systems as well as in middle and high pressure driving systems. Using the low pressure leads to low cost of the components, and to easy operation and safety driving of the systems. The studies on the low pressure water hydraulic systems have been carried out to aim to get the low price compatible to pneumatic systems while the high power density and good controllability are compatible to oil hydraulic systems [2],[3].

Water hydraulic system has been applied in industries of food processing, beverage bottling and packaging, semiconductor and paper manufacturing, etc. It has also possible applications in welfare equipments, universal house equipments, leisure and amusements park equipments and others [4]. For the many of those applications, the low pressure water hydraulic systems are available. Some of them can be driven directly by the pressure from the civil tap water network or the water supply network for the industries. Otherwise, centrifugal pumps may be often installed as pressure sources. In all the cases, pressure intensifier will be useful when the particular actuators require the higher pressure than the primary pressure from the pressure sources in the systems.

The pressure intensifiers, or boosters, convert high-flow low-pressure fluid power into low-flow high-pressure fluid power. There are many commercial products of oil hydraulic and pneumatic pressure intensifiers. Most of them are cylinder-type and there are a single acting type and a double acting type. The range of their working pressure is very wide; some works at below $0.1 \mathrm{MPa}$ supply pressure and some works with a few hundred $\mathrm{MPa}$ intensified output pressure. The intensification ratio is also in variety; $1: 2$ to $1: 80$ [5]. Besides the cylinder type, there are a few kinds of rotary type intensifier; one is commonly known as a multiple-section, internal gear flow divider which is appropriately connected into a circuit to make intensification result. Another is a liquid transformer based on a called "radial cylinder motor" [6]. It is designed for oil hydraulic driving with relatively high pressure. It is also expected to be suitable for water hydraulic driving because of its structure with low friction and small leakage. Up to the present, however, the pressure intensifiers available in low pressure water hydraulic systems are very few in market.
In this study, a new rotary type water hydraulic pressure intensifier which is available in low pressure water hydraulic systems is presented. It consists of a small number of parts and simple structure. It is formed with a little modification from a water hydraulic planetary gear motor. Its main part consists of a stator, a rotor, nine planetary gears and two side plates [7]. The object of this paper is to introduce the basic structure and the working principle of the new pressure intensifier, and to present some experimental results on fundamental characteristics of a prototype.

\section{STRUCTURE AND WORKIG PRINCEPL}

The basic structure of the pressure intensifier is the same as a water hydraulic planetary gear motor except the location of the distribute holes on the port plate A. The main part of the water hydraulic planetary gear motor consists of a stator, a rotor, nine planetary gears, the port plates A, B and a flange as shown in Figures 1 and 2 .

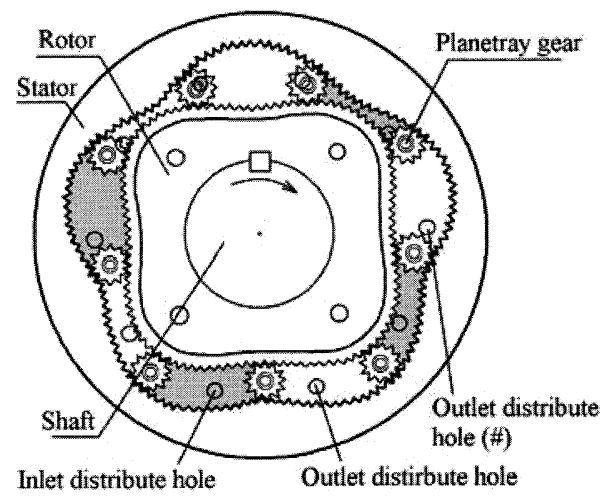

Figure 1 Structure of main part of water hydraulic planetary gear motor

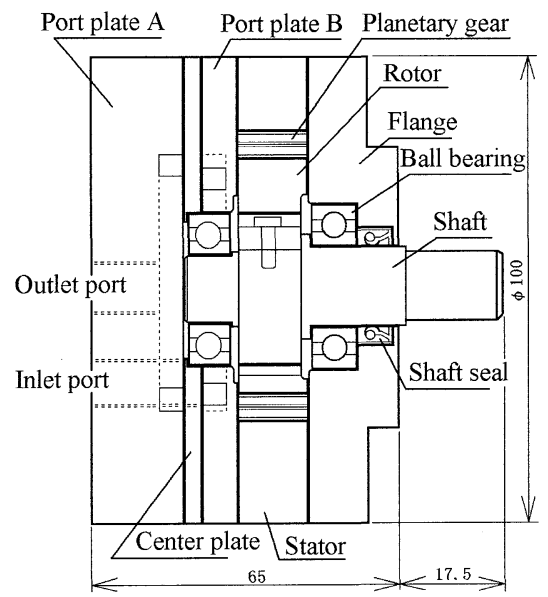

Figure 2 Cross sectional drawing of water hydraulic planetary gear motor 
The inside of the stator is formed by a curve with 5 lobes and the outside of the rotor is formed by a curve with 4 lobes. The shape and size of the pitch curves of the both of them and the radius of the planetary gear's pitch circle has a tight connection. They are all determined by numerical calculation of the equation which is derived based on the theorem of friction wheel model [8]. The curved surfaces of the stator and the rotor have teeth which mesh with the planetary gears. Nine displacement chambers enclosed by the stator, rotor, planetary gears, port plate $\mathrm{B}$ and flange are formed, and the each chamber's volume varies periodically during the rotor rotates.

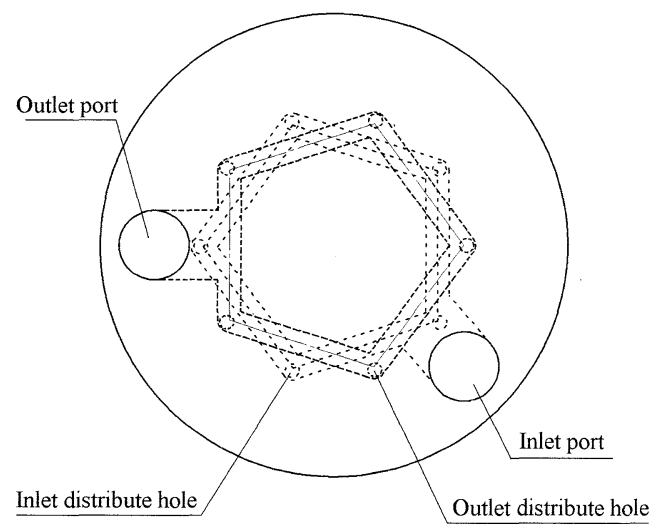

Figure 3 Location of Inlet and Outlet distribute holes on the side plate A and B for the motor

Port plate A has an outlet port and five outlet distribute holes which are all connected with a pentagon groove and port plate $\mathrm{B}$ has an inlet port and five inlet distribute holes which are all connected with a pentagon groove such as shown in Figure 3. The inlet port is also drilled through the port plate $A$ to connect the inlet port on the port plate $\mathrm{B}$, and the five outlet distribute holes are also drilled through the port plate $B$ at the same place of each the outlet distribute hole on the port plate A. The inlet distribute holes and the outlet distribute holes are located alternately on the inside surface of the port plate $\mathrm{B}$ and open to the displacement chambers as shown in Figure1. Each displacement chamber connects alternately to an inlet distribute hole and an outlet distribute hole during the rotor rotates. The volume of each displacement chamber increases while connecting to the inlet distribute hole and decreases while connecting to the outlet distribute hole. The water from the chamber is exhausted through the outlet distribute holes to the outlet port and to the reservoir.

In the displacement chambers which connect to the inlet distribute holes, the shadowed chambers in Fig.1, the pressure acts on the rotor surface and the planetary gears generates the torque in clockwise. Fig. 4 shows the mechanism of torque generation at one of the displacement chambers. The drawing of (a) shows the generation of torque by the pressure acts on the rotor surface and the drawing of (b) shows that on the planetary gears. Note that there is a distance ' $e$ ' between the line of force action and the center of the rotor in (a), and that $r_{1}$ is larger than $r_{2}$ in (b). The same condition appears at the all displacement chambers when they are connecting to the inlet distribute holes.

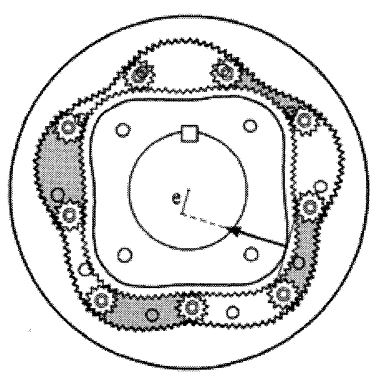

(a)

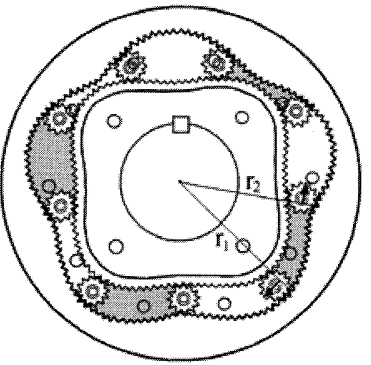

(b)
Figure 4 Mechanism of torque generation

As the planetary gears revolve with the rotation of the rotor, the displacement chambers move and switch the connection to the inlet and outlet distribute holes by turns. The rotation of the rotor, therefore, continues while pressurized water is supplied to the inlet port.

The only one modification to get the intensification function is to replace the port plate A by a modified one as shown in Figure 5. On the new port plate A, one of the outlet distribute holes is separated from the other 4 as well as the outlet port. The volume of the displacement chamber decreases while it connects to the separated outlet distribute hole. The displacement chamber works as a pump which is driven by the water hydraulic planetary gear motor, and the chamber therefore discharges intensified higher pressure water to the intensified port in Fig.5, which is equivalent to the outlet distribute hole (\#) in Figure 1.

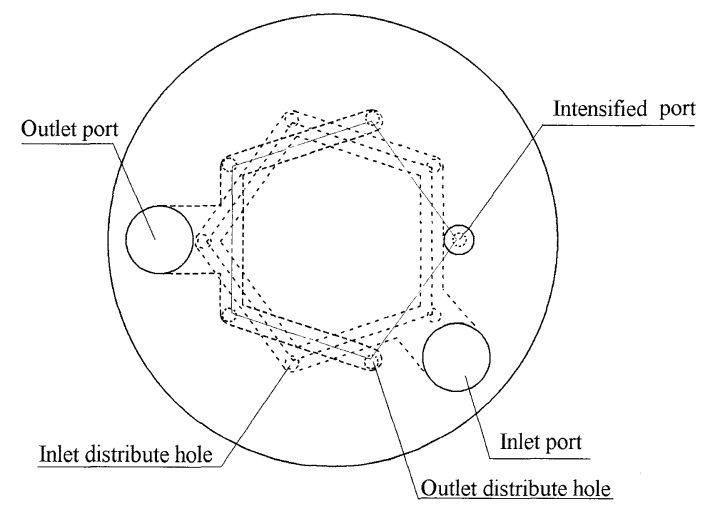

Figure 5 Modified port plate A 


\section{BASIC THEORY}

The torque $T_{s}$ generated by the supply pressure $P_{S}$, which tends to rotate the rotor, balances with the counter torques $T_{i}$ and $T_{e}$ generated by pressures $P_{i}$ and $P_{e}$. The torque balance is presented by Eq.(1).

$$
T_{s}=T_{i}+T_{e}
$$

Here, the torques are presented as $T_{s}=\eta_{T s} \frac{P_{s} \cdot V_{t h}}{2 \pi}$, $T_{i}=\frac{1}{\eta_{T i}} \frac{P_{i}}{2 \pi} \frac{V_{t h}}{n}$ and $T_{e}=\frac{1}{\eta_{T e}} \frac{P_{e}}{2 \pi} \frac{(n-1) \cdot V_{t h}}{n}$.

Substituting them into Eq.(1), the following equation is derived.

$$
P_{i}=n \cdot \eta_{T s} \cdot \eta_{T i} \cdot P_{s}-\frac{\eta_{T i}}{\eta_{T e}}(n-1) \cdot P_{e}
$$

Supposing $P_{e}=0$ and putting $\eta_{T s} \cdot \eta_{T i}=\eta_{T}$ in Eq.(2), the intensified pressure is presented by Eq.(3).

$$
P_{i}=\eta_{T} \cdot n \cdot P_{s}
$$

Concerning the flow rate, the supply flow rate $Q_{s}$, the intensified port flow rate $Q_{i}$ and the exhausted flow rate from the outlet port $Q_{e}$ are presented as follows,

$$
Q_{s}=\frac{N \cdot V_{t h}}{\eta_{V s}}, Q_{i}=\eta_{V i} \cdot N \cdot \frac{V_{t h}}{n} \text { and } Q_{e}=Q_{s}-Q_{i} .
$$

Putting $\eta_{V s} \cdot \eta_{V i}=\eta_{V}$, the intensified port flow rate is presented by Eq.(4).

$$
Q_{i}=\eta_{V} \frac{Q_{s}}{n}
$$

The total efficiency is defined by Eq.(5).

$$
\eta=\frac{P_{i} \cdot Q_{i}}{P_{s} \cdot Q_{s}}
$$

The following relation is confirmed by substitution of Equations (3) and (4) to the Eq.(5).

$$
\eta=\eta_{T} \cdot \eta_{V}
$$

\section{SPECIFICATION OF A PROTOTYPE}

Basically the prototype is made by a modification of a water hydraulic planetary gear motor, changing the port plate A. The external sizes are shown in Fig.1. The theoretical displacement volume $V_{\text {th }}$ is $37.6 \mathrm{~cm}^{3} / \mathrm{rev}$. The numbers of lobe are 4 with the rotor and 5 with the stator, and therefore the numbers of the inlet distribute holes and the outlet distribute holes are 5 respectively. The pitch curve of the rotor is formed by cosine curve of which the maximum radius is $27.524 \mathrm{~mm}$ and the minimum radius is $22.552 \mathrm{~mm}$. The width of the rotor, stator and planetary gears are $15 \mathrm{~mm}$. All the main parts are made of stainless steel and the surfaces of the port plate B and the flange are coated with DLC treatment. The tentative specification of the prototype of the water hydraulic planetary gear motor is presented in Table 1 . Fig.6 shows the inside view of the motor.

Table 1 Tentative specification of the prototype of the water hydraulic planetary gear motor

\begin{tabular}{|l|l|}
\hline Working fluid & tap water \\
\hline Rated supply pressure & $0.25 \mathrm{MPa}$ \\
\hline Rated supply flow rate & $0.008 \mathrm{~m}^{3} / \mathrm{min}$ \\
\hline Rated rotational speed & $200 \mathrm{rpm}$ \\
\hline Rated outpet torque & $0.9 \mathrm{Nm}$ \\
\hline Rated output power & $20 \mathrm{~W}$ \\
\hline
\end{tabular}

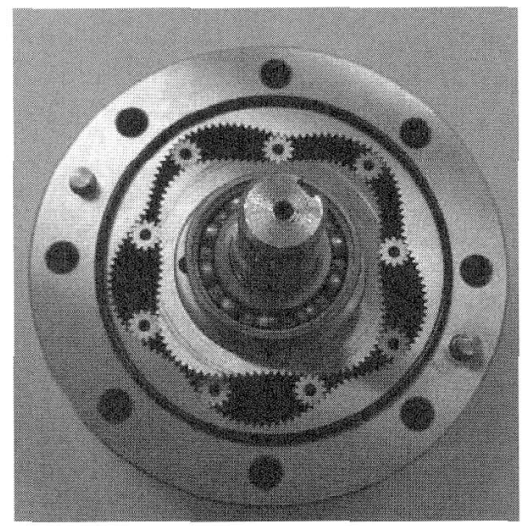

Figure 6 Inside view of the prototype of the water hydraulic planetary gear motor

According to the Eq.(3), the intensification ratio is presented by Eq.(7) for the prototype because the number of the distribute holes $n$ is 5 . The intensification ratio will be the maximum 5 when $\eta_{T}=1$.

$$
\frac{P_{i}}{P_{s}}=\eta_{T} \times 5
$$

\section{EXPERIMENTAL RESULTS}

An experiment is carried out in order to confirm the fundamental function as a pressure intensifier and how large the critical intensification ratio is. Fig. 7 shows the circuit of the experimental setup.

Water is supplied from the axial piston pump and the 
supply pressure $P_{s}$ is set at a given value using the relief valve and the throttle valve (1). The intensified pressure $P_{i}$ is set at an optional value using the throttle valve (2). The pressures $P_{s}, P_{i}$ and $P_{e}$ are measured with Bourdon pressure gauges which contain glycerin as damper oil. The flow rate $Q_{s}$ and $Q_{i}$ are measured with turbine flow meters and the rotational speed of the rotor shaft is measured with a digital tachometer.

The flow from the intensified port is discharged periodically and intermittently. The condition is almost the same as in the case of the double acting cylinder type intensifier. There is fluctuation on the intensified port pressure and the fluctuation becomes especially large when the rotational speed is low. An accumulator of $30 \mathrm{~cm}^{3}$ is installed at the intensified port line to reduce the pressure fluctuation.

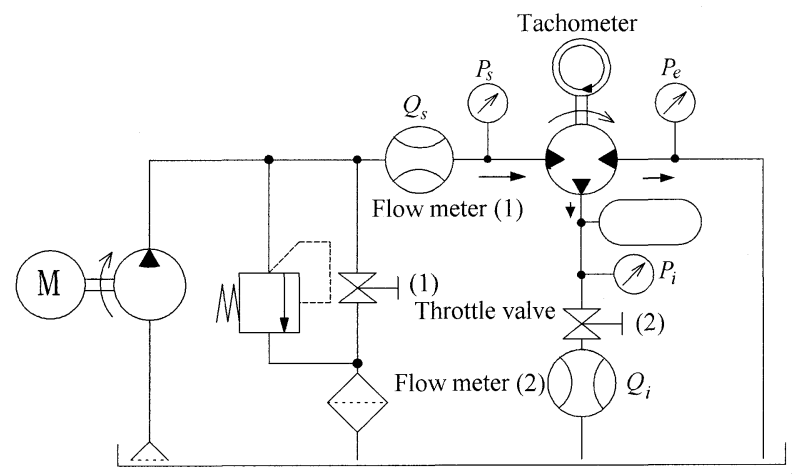

Figure 7 Circuit of the experimental setup

Fig. 8 shows the experimental result of the flow rate from the intensified port $Q_{i}$ versus the intensification ratio $P_{i} / P_{s}$ at the five different supply pressures. The maximum values of $P_{i} / P_{s}$ are between 2.5 and 2.7 at the each supply pressure. When the ratio $P_{i} / P_{s}$ is larger than the maximum values, the rotation of the shaft becomes intermittently and the intensified pressure fluctuates violently. Therefore, there is no plot over the area. The maximum value of $P_{i} / P_{s}$ is the critical intensification ratio.

It is confirmed that the new structured intensifier works well as a water hydraulic pressure intensifier even at a low pressure near the pressure of the civil tap water network.

It is found from Fig. 8 that $Q_{i}$ decreases as the ratio $P_{i} / P_{s}$ becomes larger under a constant $P_{s}$, and $Q_{i}$ increases as $P_{s}$ becomes higher under a constant $P_{i} / P_{s}$. This tendency is caused by the change in the rotational speed of the rotor. Fig.9 shows the rotational speed $N$ versus $P_{i}$ $/ P_{s}$. The curves are almost the same as in Fig.8.

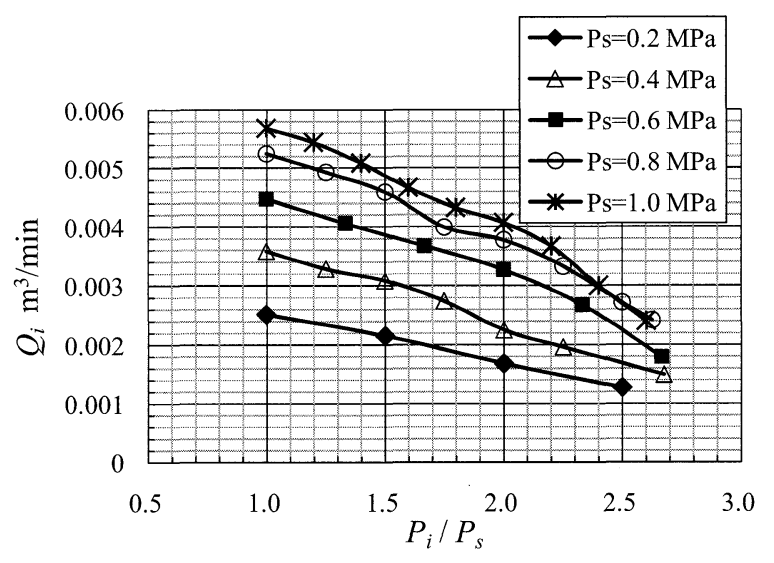

Figure 8 Flow rate from the intensified port $Q_{i}$ versus the intensification ratio $P_{i} / P_{s}$

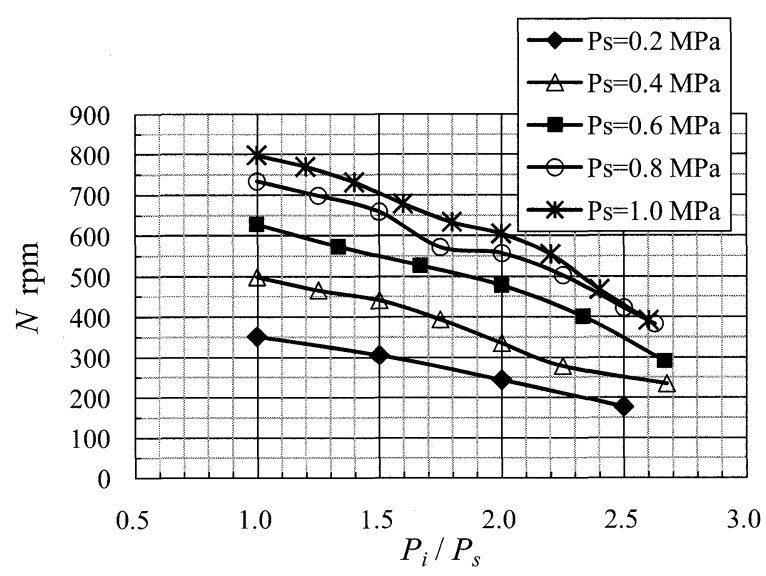

Figure 9 Rotational speed $N$ versus the intensification ratio $P_{i} / P_{S}$

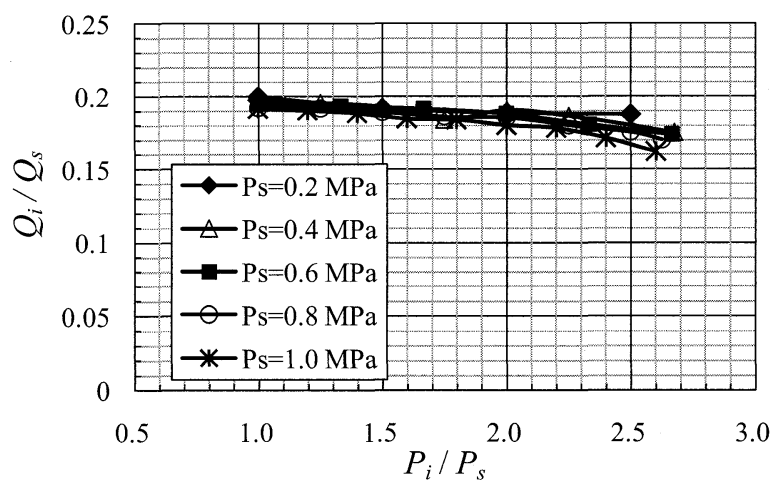

Figure 10 Ratio of $Q_{i}$ to the supply flow rate $Q_{s}$ versus the intensification ratio $P_{i} / P_{s}$

The ratio of $Q_{i}$ to the supply flow rate $Q_{s}$ versus $P_{i} / P_{s}$ is shown in Fig.10. If there is no leakage, that is $\eta_{\nu}=1$, the ratio $Q_{i} / Q_{s}$ becomes 0.2 as shown in Eq.(4). Since the 
leakage increases as $P_{s}$ becomes higher and the ratio $P_{i}$ $/ P_{s}$ becomes larger, the value of $Q_{i} / Q_{s}$ becomes smaller than 0.2 . Fig. 11 shows the same result by the volumetric efficiency $\eta_{v}$.

Fig.12 shows the torque efficiency $\eta_{T}$ versus $P_{i} / P_{s}$. The value of $\eta_{T}$ is given by the ratio $P_{i} / P_{S}$ as shown in

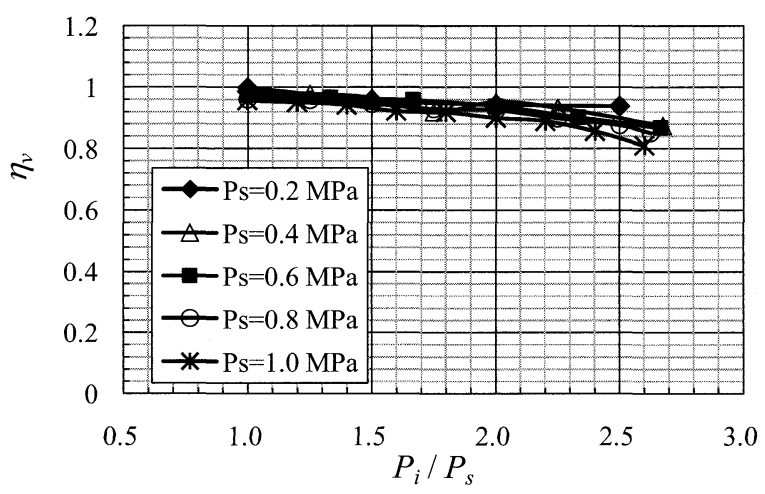

Figure 11 Volumetric efficiency $\eta_{v}$ versus the intensification ratio $P_{i} / P_{s}$

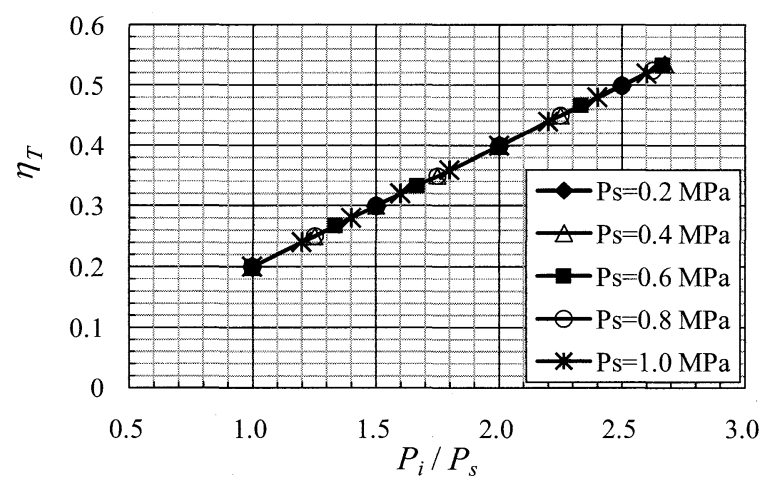

Figure 12 Torque efficiency $\eta_{T}$ versus the intensification ratio $P_{i} / P_{s}$

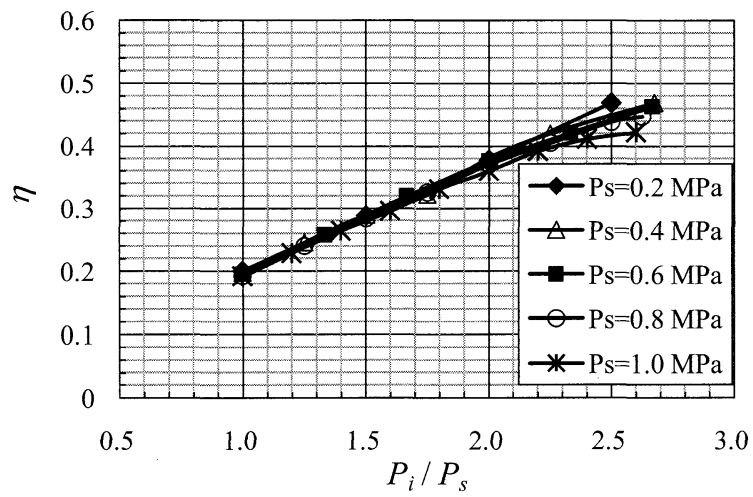

Figure 13 Total efficiency $\eta$ versus the intensification ratio $P_{i} / P_{s}$
Eq.(7) regardless of the value of $P_{s}$. The maximum value of $P_{i} / P_{s}$ is obtained when $\eta_{T}$ is the maximum. In order to get a larger intensification ratio, to improve the torque efficiency is the key.

Fig. 13 shows the total efficiency $\eta$ versus $P_{i} / P_{s}$. The maximum total efficiency is around $45 \%$. It is found that $\eta$ becomes higher as $P_{i} / P_{s}$ is larger because of increase in torque efficiency $\eta_{T}$. And it is also found that $\eta$ decreases as the supply pressure $P_{s}$ becomes higher under the condition of constant $P_{i} / P_{s}$. It is caused by decrease in volumetric efficiency $\eta_{v}$. For the further improvement of the total efficiency and to get a larger intensification ratio, the improvement in torque efficiency is important.

\section{CONCLUSION}

It is confirmed that a new rotary type pressure intensifier, which is constructed by a simple modification with a water hydraulic planetary gear motor, works well as a pressure intensifier. It works even in low pressure as same as the pressure in civil tap water network. The critical intensification ratio of the prototype is around 2.5 and the total efficiency is about $45 \%$. The key to increase the total efficiency and to get the larger intensification ration is to improve the torque efficiency.

\section{REFERENCES}

1. Modern Water Hydraulics - Your Choice for the Future, Leaflet of Introduction of Water Hydraulics by NFPA, 1995.

2. Aaltonen, J. et al., Experiences On The Low Pressure Water Hydraulic Systems, Proceedings of the Fourth JFPS International Symposium, 1999, pp.357-363.

3. Kunttu, P. et al., Low Pressure Water Hydraulics Stae of the Art, Proceedings of The Sixth Scandinavian International Conference on Fluid Power, 1999, pp.67-75.

4. Aqua Drive System - A Technical Guide 2, Leaflet of Introduction of Water Hydraulics by JFPA, 2005.

5. A staff report, Intensifiers offer boost from low to high pressure, Hydraulics \& Pneumatics, 1999, 52-7, pp.45-46.

6. Yasuo Kita, Takashi Asami et al., Rotary type liquid pressure transformer, Journal of JFPS Fluid Power System (in Japanese), 2004, 35-1, pp.67-71.

7. Shigeru Oshima, et at., Development of a Low Pressure Water Hydraulic Planetary Gear Motor, Proceedings of JSME annual conference 2006 (in Japanese), 2006, 2, pp.327-328.

8. J.W.G.M. Huijbers, Kinematics of the Planetary Gear Motor, Report PGM-T1, Hessels \& van Rooij Engineering, 1990. 